\title{
Antimicrobial resistance of pathogens causing nosocomial bloodstream infection in Hubei Province, China, from 2014 to 2016: a multicenter retrospective study
}

Lei Tian', Ziyong Sun ${ }^{1}$ and Zhen Zhang ${ }^{2 *}$

\begin{abstract}
Background: Data on the pathogens responsible for nosocomial bloodstream infection (BSI) and their antimicrobial resistance (AMR) in Hubei province are limited. This study was conducted to determine the major pathogens causing BSI and to characterize their AMR.

Methods: Data from the China Antimicrobial Resistance Surveillance System (CARSS) from 2014 to 2016 were analyzed retrospectively.

Results: Escherichia coli, Staphylococcus aureus and Klebsiella pneumoniae were the most common pathogens responsible for nosocomial BSI. Individuals aged $0-5$ years and $\geq 40$ years old were the major demographics at risk of infection by E. coli, K. pneumoniae, Pseudomonas aeruginosa, Acinetobacter baumannii and Enterobacter cloacae, while individuals aged $0-5$ years were the major demographic at risk of infection by $S$. aureus, Enterococcus faecalis, E. faecium, Streptococcus pneumoniae and Stenotrophomonas maltophilia. The frequencies of E. coli and $K$. pneumoniae isolates resistant to cefotaxime were $59.1 \%$ and $24.3 \%$, respectively, and the frequencies of resistant isolates to ceftazidime were $42.9 \%$ and 27.2\%, respectively. From 2014 to 2016, the frequency of extended-spectrum B-lactamase (ESBL)-positive E. coli declined from 29.07 to 24.5\%, and the frequency of ESBL-positive K. pneumoniae declined from 18.64 to $12.33 \%$. The frequency of carbapenem-resistant (CR) E. coli was below $0.5 \%$, but $1-10 \%$ of $K$. pneumoniae isolates were CR.
\end{abstract}

Conclusions: The emergence of methicillin-resistant S. aureus and the expansion of ESBL and fluoroquinolone resistance among Gram-negative Enterobacteriaceae increased AMR severity. Carbapenemase-producing K. pneumoniae isolates responsible for nosocomial BSI increased year over year and effective infection control measures should be taken to prevent them from spreading.

Keywords: Nosocomial bloodstream infection, Antimicrobial resistance, MRSA, ESBL

\section{Background}

Bloodstream infection (BSI) is very common and is associated with high morbidity and mortality worldwide. The etiology of BSI and the antimicrobial resistance (AMR) of the pathogens causing it differ significantly between developed and developing countries [1]. Data from the

\footnotetext{
* Correspondence: 258452160@qq.com

${ }^{2}$ Department of Pharmacy, Tongji Hospital, Tongji Medical College, Huazhong University of Science and Technology, Wuhan, Hubei Province, China

Full list of author information is available at the end of the article
}

Finnish Hospital Infection Program during 1999-2001 and 2005-2010 demonstrated that the most common pathogens responsible for BSI were coagulase-negative Staphylococcus, S. aureus and Escherichia coli [2]. By contrast, BSI surveillance in Malawi from 1998 to 2016 indicated that non-typhoidal Salmonella, S. enterica Typhi and Streptococcus pneumoniae were the most common causative agents [3]. In China, the pathogens responsible for BSI vary from region to region. A study of the clinical features of nosocomial BSI in neonates from two prefectures, Henan and Chongqing, indicated

(c) The Author(s). 2018 Open Access This article is distributed under the terms of the Creative Commons Attribution 4.0 International License (http://creativecommons.org/licenses/by/4.0/), which permits unrestricted use, distribution, and reproduction in any medium, provided you give appropriate credit to the original author(s) and the source, provide a link to the Creative Commons license, and indicate if changes were made. The Creative Commons Public Domain Dedication waiver (http://creativecommons.org/publicdomain/zero/1.0/) applies to the data made available in this article, unless otherwise stated. 
that E. coli was the most prevalent BSI pathogen in Henan while Klebsiella pneumoniae was the predominant BSI pathogen in Chongqing [4]. Reports on the causes of BSI in Hubei province have been limited.

Surveillance and analysis of AMR are crucial in preventing BSI. The Hubei Province bacterial resistance surveillance network was founded in 1998 and includes surveillance data on hospital-acquired and community-acquired infections. By the end of 2016, a total of 11 secondary care hospitals and 41 tertiary care hospitals had joined the network, covering all prefecture-level cities in Hubei Province (Fig. 1). Here, in order to provide a basis for empirical treatment and policy making for the Department of Health Administration, we reported BSI surveillance data over the last three years, from 2014 to 2016.

\section{Methods}

\section{Study design and data collection}

We undertook a retrospective surveillance study of nosocomial BSI in Hubei province. The data were derived from the China Antimicrobial Resistance Surveillance System (CARSS). Hospital-acquired, nosocomial, and health care-associated infections were defined according to the World Health Organization (WHO) as infections occurring in a patient residing in a hospital or another health care facility, in whom the infection was not present nor incubating at the time of admission [5]. An infection occurring $48 \mathrm{~h}$ or later after admission to hospital was considered nosocomial [5]. An infection occurring within $48 \mathrm{~h}$ of admission to hospital or not associated with healthcare interventions within the previous 12 months was defined as community-acquired [6]. If the isolates were identified as coagulase-negative Staphylococcus, Corynebacterium, Bacillus, Propionibacterium or other potential skin contaminants, two or more separate blood cultures were required for diagnosis [7].

\section{Strain identification and antibiotic sensitivity testing}

Blood samples $(8-10 \mathrm{~mL}$ for adult patients and $2-5 \mathrm{~mL}$ for pediatric patients) were collected into special blood culture bottles. Various automated blood culture instruments were applied. Manual biochemical tests, automatic bacteria identification instruments and/or an IVD-MALDI Biotyper (Bruker, Germany) were used for identification of bacterial species. The Kirby-Bauer method and/or the minimum inhibitory concentration (MIC) method were used for antimicrobial susceptibility testing according to the Clinical and Laboratory Standards Institute (CLSI) guidelines. The results of antimicrobial sensitivity tests were interpreted according to the CLSI 2016 standards [8]. Isolation and identification of strains and antibiotic sensitivity testing were performed by members of the bacterial resistance monitoring network. Each member of the monitoring network reported data regarding bacterial identification and antibiotic sensitivity to the CARSS each quarter.

\section{Statistical analysis}

Antibiotic susceptibility data was analyzed using WHONET 5.6 software. Only the first isolate of a given species from a patient was analyzed according to CLSI M-39 [9]. When antibiotic resistance tests were carried out by the Kirby-Bauer method, the MIC method and the E-test method simultaneously, the results of E-tests

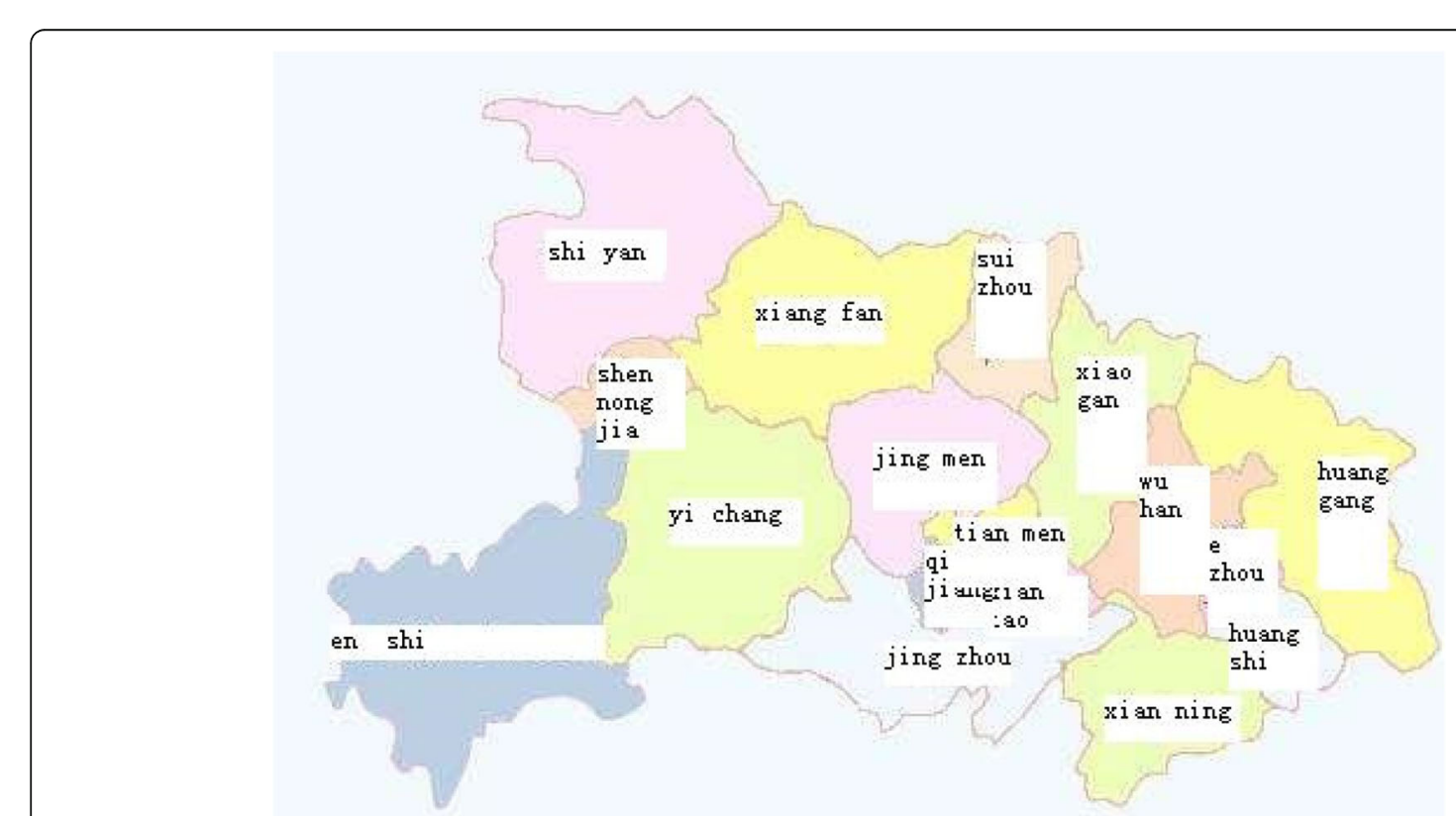

Fig. 1 Location distribution of the members of the monitoring network 
were preferred, followed by the MIC method and the Kirby-Bauer method.

\section{Results}

\section{Distribution of common pathogens responsible for BSI}

In this investigation, only strains isolated more than 100 times per year were analyzed. E. coli was the most common pathogen responsible for nosocomial BSI during 2014-2016, followed by S. aureus and K. pneumonia. Each of these pathogens was isolated more than 500 times per year (Fig. 2a). Enterococcus faecium, E. faecalis, Pseudomonas aeruginosa and Acinetobacter Baumannii isolated were each isolated between 250 and 500 times per year (Fig. 2b). Enterobacter cloacae, S. pneumoniae and

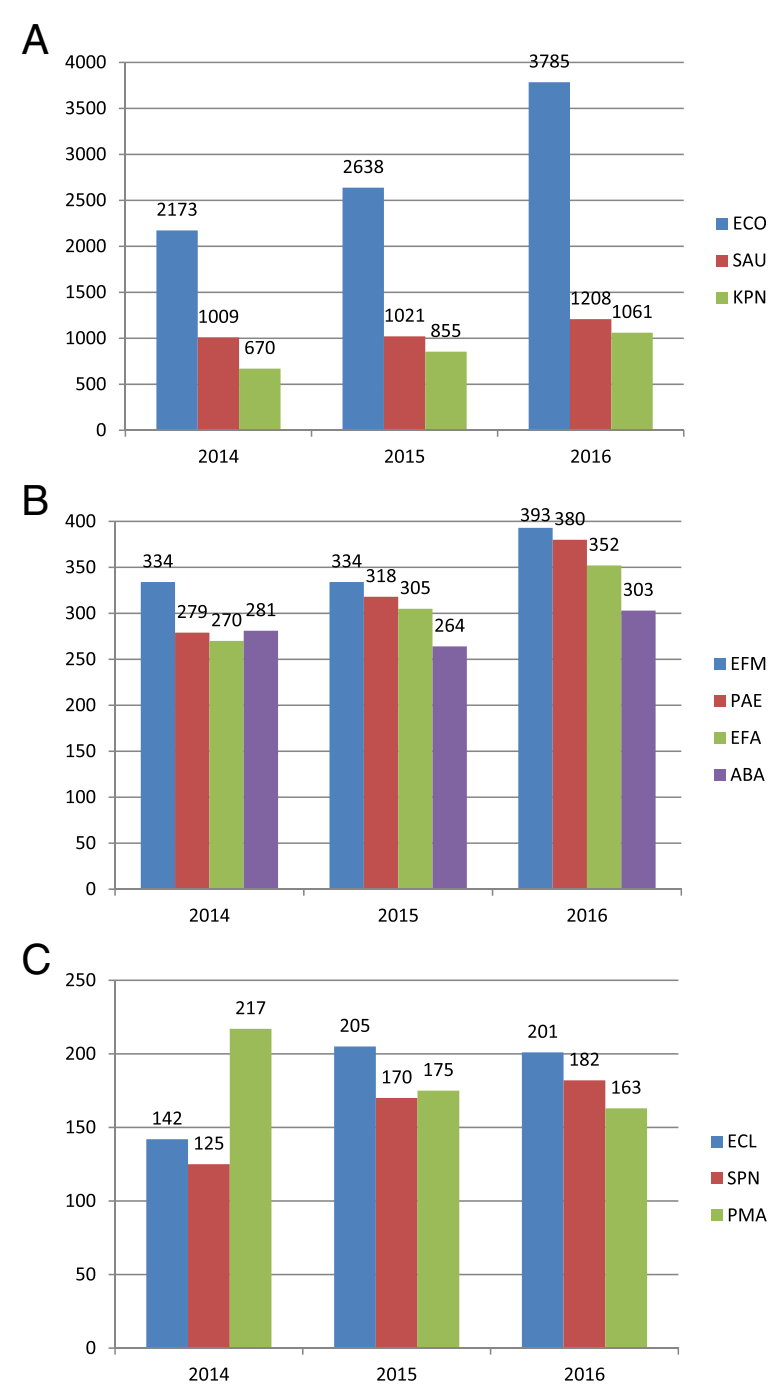

Fig. 2 Pathogens in bloodstream infection, 2014 2016. a Pathogens isolated at high frequency ( $N>500 /$ year). b Pathogens isolated at intermediate frequency (250/year $<N<500 /$ year). c Pathogens isolated at low frequency (100/year $<N<250 /$ year)
Stenotrophomonas maltophilia were each isolated between 100 and 250 times per year (Fig. 2c).

\section{Age demographics affected by BSI}

Individuals aged $0-5$ years and $\geq 40$ years old were the major demographics at risk of infection by $E$. coli, $K$. pneumoniae, $P$. aeruginosa, $A$. baumannii and $E$. cloacae, while individuals aged $0-5$ years were the major demographic at risk of infection by $S$. aureus, E. faecalis, E. faecium, S. pneumoniae and S. maltophilia (Fig. 3).

\section{Seasonal distribution of BSI pathogens}

Most E. coli isolates were identified from October to December (10-12) and from July to September (7-9), with the lowest number identified from January to March (1-3). Isolates of S. aureus, E. faecalis and E. faecium were distributed roughly evenly across all four seasons. The highest number of $K$. pneumoniae, $P$. aeruginosa, A. baumannii and $E$. cloacae isolates identified in a quarter was 7-9 and the lowest number of these species was 1-3. Most $S$. maltophilia isolates were identified from April to June (4-6) and the lowest number observed in a quarter was 1-3 (Fig. 4).

\section{Antimicrobial susceptibility}

For E. coli isolates, resistance rates to ampicillin (AMP), piperacillin (PIP), cefazolin (CZO), cefuroxime (CXM), cefotaxime (CTX) and trimethoprim sulfamethoxazole (SXT) were $>50 \%$, but more than $89 \%$ of isolates were sensitive to imipenem (IPM), meropenem (MEM), piperacillin-tazobactam (TZP), amikacin (AMK) and cefoxitin (FOX) (Fig. 5a). K. pneumoniae isolates were naturally resistant to AMP, and resistance rates to all other antibiotics except for PIP were > $50 \%$ (Fig. 5b). E. cloacae isolates were naturally resistant to AMP, PIP, CZO, CXM, FOX and ampicillin-sulbactam (SAM), but $>50 \%$ of isolates were sensitive to the other antibiotics (Fig. 5c). P. aeruginosa isolates were naturally resistant to SXT, and the resistance rate to minocycline (MNO) was $80.4 \%$. However, $>60 \%$ of isolates were sensitive the other antibiotics except for ticarcillin-clavulanate Acid (TCC) (Fig. 5d). More than $65 \%$ of $A$. baumannii isolates were resistant to common antibiotics except for MNO (Fig. 5e). Sensitivity rates of S. maltophilia isolates to SXT, MNO, levofloxacin (LVX) and chloramphenicol (CHL) exceeded $80 \%$ (Fig. 5f). More than $50 \%$ of $S$. aureus isolates were resistant to penicillin (PEN), erythromycin (ERY), CZO and CXM (Fig. 5g). More than $60 \%$ of $E$. faecalis isolates were sensitive to common antibiotics except for ERY and ciprofloxacin (CIP) (Fig. 5h). For E. faecium isolates, sensitivity rates to vancomycin (VAN), teicoplanin (TEC) and linezolid (LNZ) exceeded 95\%, but resistance rates to other antibiotics exceeded 60\% (Fig. 5i). For S. pneumoniae isolates, resistance rates to ERY and clindamycin (CLI) exceeded $89 \%$, but sensitivity rates to other antibiotics exceeded 80\% (Fig. 5j). 


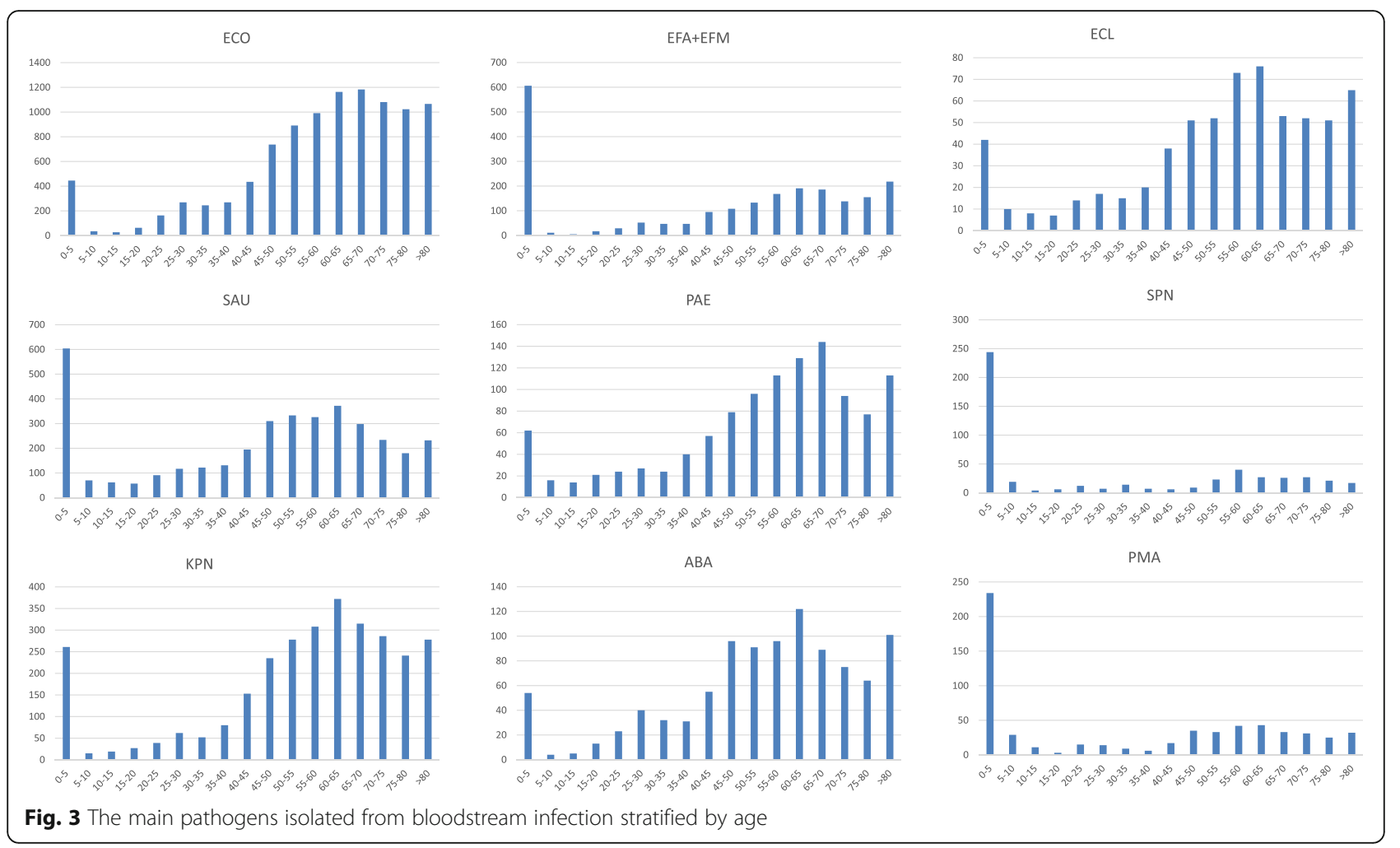

Trends of multi-drug resistant (MDR) strains

The frequency of methicillin-resistant $S$. aureus (MRSA), extended-spectrum $\beta$-lactamase (ESBL)-positive $E$. coli and $K$. pneumoniae, and carbapenem-resistant (CR) E. coli and K. pneumoniae were analyzed during 2014-2016. The proportion of MRSA isolates ranged from 30 to $40 \%$, ESBL-positive $E$. coli isolates ranged from 20 to $30 \%$, and ESBL-positive $K$. pneumoniae isolates ranged from 10 to $20 \%$. The frequency of CR-E. coli was below $0.5 \%$, but CR- $K$.

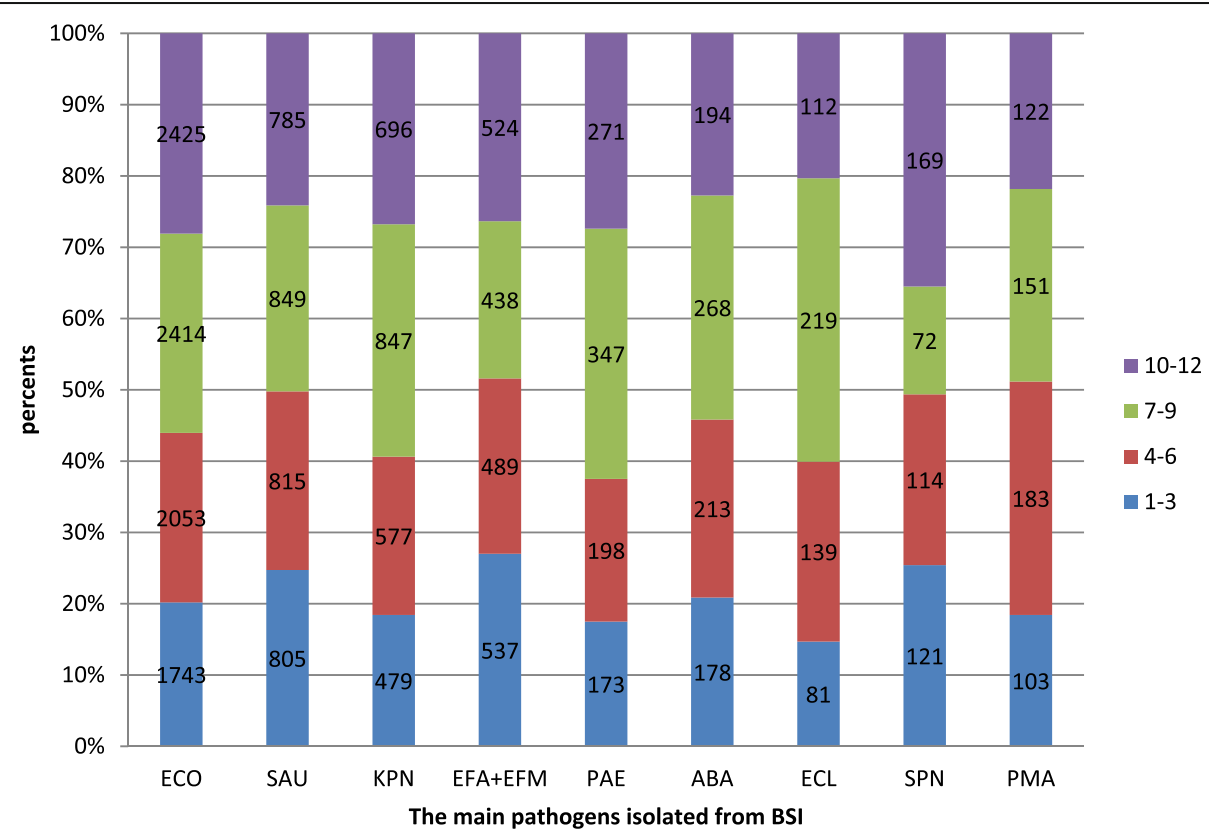

Fig. 4 The distribution proportion in the four seasons of the main pathogens isolated from BSI 

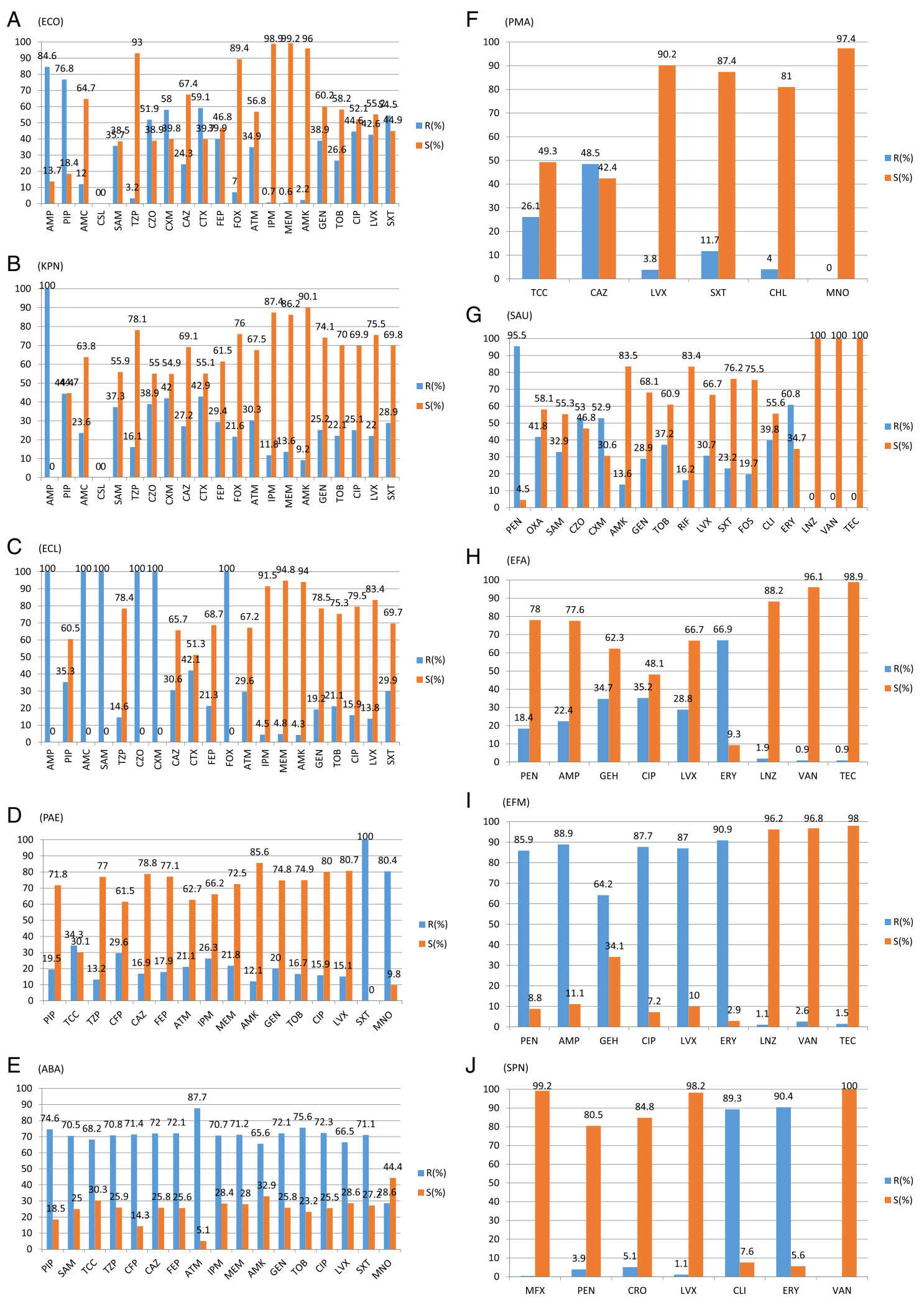

Fig. 5 Susceptibility rates and resistance rates to common antibiotics. a (Escherichia coli). b (Klebsiella pneumoniae). c (Enterobacter cloacae). d (Pseudomonas aeruginosa). e (Acinetobacter Bauman). f (Stenotrophomonas maltophilia). $\mathbf{g}$ (Staphylococcus aureus). $\mathbf{h}$ (Enterococcus faecalis). $\mathbf{i}$ (Enterococcus faecium). $\mathbf{j}$ (Streptococcus pneumonia) 
pneumoniae isolates were detected at a frequency of $1-10 \%$ (Fig. 6).

\section{Discussion}

Our surveillance data indicated E. coli, S. aureus and K. pneumoniae were the most common pathogens responsible for nosocomial BSI in Hubei province from 2014 to 2016. For common gram-positive bacteria and S. maltophilia, individuals $<5$ years of age were the major demographic at risk of nosocomial BSI. By contrast, for common gram-negative bacteria except S. maltophilia, individuals $<5$ years of age and $\geq 40$ years of age were the main demographics at risk of BSI. Resistance of $E$. coli isolates to common antibiotics was more severe than $K$. pneumoniae isolates, especially to third-generation cephalosporins and fluoroquinolones. ESBL production by $E$. coli isolates was more common than in K. pneumoniae isolates. Most $P$. aeruginosa isolates $(>60 \%)$ were sensitive to antibiotics, while most $A$. baumannii isolates (>60\%) were antibiotic-resistant.

E. coli, S. aureus and K. pneumoniae were the most common pathogens responsible for BSI according to our surveillance data. These results differed from reports in European and African populations, in which coagulase -negative Staphylococcus and non-typhoidal Salmonella were the predominant causative agents of BSI (in Finland and Malawi, respectively) [2, 3]. This may be due to the distinct social, economic and environmental factors in these regions. The spectrum of pathogens responsible for BSI is highly variable both geographically and temporally [10]. Timely analysis and reporting of local AMR resistant isolates is important for infection control.

The most common pathogens responsible for BSI are different in people of different ages [3]. Our study found that individuals aged $0-5$ years old were an important demographic at high risk of BSI. It has been well established that children under 5 years of age are susceptible to infection by Salmonella and other intestinal

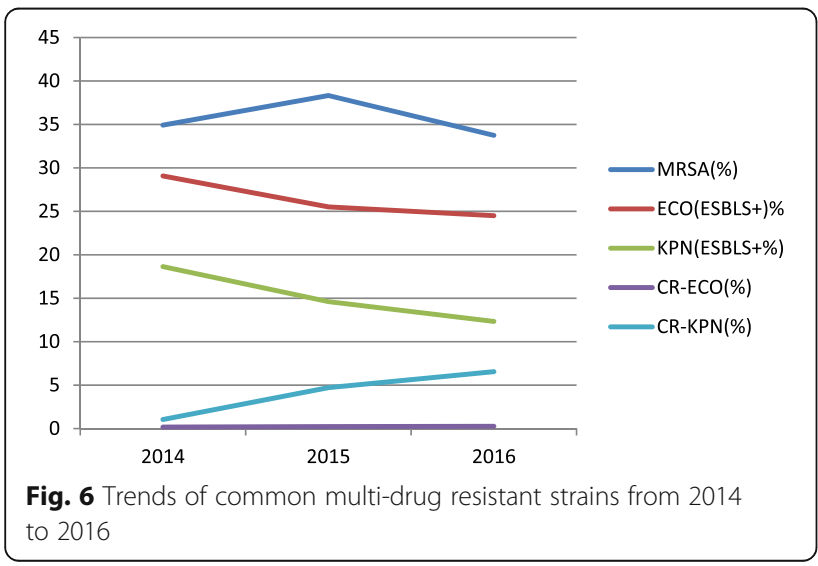

pathogens [11]. Our findings indicated that children under 5 years of age were also the main demographic susceptible to BSI. For Gram-positive bacteria (S. aureus, E. faecalis, E. faecium, and S. pneumoniae) and S. maltophilia, individuals under 5 years of age were the major demographic at risk, while individuals above 40 years of age or under 5 years of age were the primary population at risk of infection by Gram-negative bacteria (E. coli, $K$. pneumoniae, E cloacae, P. aeruginosa and A. baumanni). Our findings indicated that the incidence of $E$. coli and K. pneumoniae BSIs was highest in elderly patients older than 40 years, consistent with global data [12]. The prolongation of human life and the aging of populations in society maybe have something to do with increasing rates of BSI caused by E. coli and K. pneumoniae.

It was reported that BSI by Bacillus cereus had a seasonal tendency with increased risk of infection during the summer [13]. Our data showed that S. pneumoniae was more often isolated in winter while $K$. pneumoniae, $P$. aeruginosa, A. baumanni, and E. cloacae were most prevalent in autumn. No obvious seasonal trend was observed for S. aureus, E. faecalis and E. faecium. Whether BSIs have seasonal trends like food-borne diseases is worth further study.

The frequency of ESBL-producing E. coli and K. pneumoniae isolates has been increasing worldwide, resulting in prolonged hospital stays, increased costs, reduced rates of clinical and microbiological responses, and poor outcomes for patients and healthcare systems [14-20]. Previous studies have analyzed the relationship between mortality rate and ESBL-producing pathogens and observed an association with KP-BSIs, although this finding remains controversial [16, 21-23]. The frequency of ESBL-producing E. coli and K. pneumoniae isolates decreased year by year from 2014 to 2016 according to our surveillance data. This finding may be related to the strategies implemented by the USA CDC for controlling the transmission of MDR bacteria such as quarantine of patients with MDR infections, strict hand disinfection by medical staff, routine disinfection of medical devices and limited use of specific antibiotics. For $E$. coli, resistances rate to the third-generation cephalosporins CTX and CAZ were $59.1 \%$ and $24.3 \%$, respectively; ESBL may be the main cause of resistance, especially for bla(CTX-M) isolates. The molecular epidemiology of E. coli isolates causing BSI in Shanghai during 2011-2013 demonstrated that CTX-14, CTX-55 and CTX-15 were the most common $\beta$-lactamases [24]. By contrast, bla(SHV) and bla(CTX-M) were the most common $\beta$-lactamases in K. pneumoniae isolates [25]. MDR and the increase in infections caused by Gram-negative bacilli producing ESBL have contributed to intensive use of carbapenems [26]. Data from the European Survey on Carbapenemase-Producing Enterobacteriaceae in Europe (EuSCAPE) indicated that 
the incidence of carbapenemase-producing (CP) E. coli and K. pneumoniae $(\mathrm{CP}-\mathrm{E} / \mathrm{K})$ isolates in hospitals increased from 0.124 per 1000 admissions in 2013 to 0.223 per 1000 admissions in 2014 [27]. Our data showed that CP-E/K isolates are a growing risk, especially CP-K. pneumoniae.

In our surveillance data, $S$. aureus was the most frequently observed Gram-positive pathogen responsible for BSI. MRSA is a worldwide issue associated with significant morbidity and mortality [28]. Our data showed that the frequency of methicillin resistance among $S$. aureus isolates was $30-40 \%$,consistent with a study conducted in 26 Hong Kong public hospitals between January 2010 and December 2012 [29], In the United States and Europe, multiple infection control approaches have been tested to control the spread of MRSA. These approaches included strict contact precautions including single rooms for MRSA-colonized or -infected patients, targeted admission screening for high-risk patients and healthcare workers at risk for infection, molecular typing of all MRSA strains, and routine decolonization of MRSA carriers including healthcare workers [28, 30, 31]. Measures to control MRSA transmission should be carried out strictly according to the principles of infection control.

This study had several limitations. In our surveillance data, only nosocomial BSI was analyzed and community-acquired BSI was not included. In Hubei province, the characteristics of community-acquired BSI are mostly unknown. The molecular mechanisms of MDR, especially ESBLs and carbapenemases, have not been tested. Molecular epidemiological studies need be carried out in the future.

\section{Conclusions}

Our findings indicated that E. coli, S. aureus and $K$. pneumoniae were the most common pathogens responsible for nosocomial BSI in Hubei province. Individuals aged $0-5$ years were the major demographic at risk of infection by S. aureus, E. faecalis, E. faecium, S. pneumoniae and $S$. maltophilia, while individuals $<5$ years of age or $\geq 40$ years of age were primarily at risk for $E$. coli, K. pneumoniae, $P$. aeruginosa, A. baumanni and E. cloacae infection. Antibiotic resistance testing showed that MDR isolates such as MRSA and ESBL-produced E. coli and $K$. pneumoniae species were still a serious concern. It was particularly noteworthy that the frequency of CR-KPN isolates was increasing significantly over time.

\footnotetext{
Abbreviations

ABA: Acinetobacter Bauman; AMK: Amikacin; AMP: Ampicillin; AMR: Antimicrobial resistance; BSI: Bloodstream infection; CARSS: China Antimicrobial Resistance Surveillance System; CHL: Chloramphenicol; CIP: Ciprofloxacin; CLI: Clindamycin; CLSI: Clinical and Laboratory Standards Institute; CP: carbapenemase-producing; CP-E/K: Carbapenemase-producing E. coli and K. pneumoniae; CR: Carbapenem-resistant; CTX: Cefotaxime; CXM: Cefuroxime; CZO: Cefazolin; ECL: Enterobacter cloacae; ECO: Escherichia coli; EFA: Enterococcus faecalis; EFM: Enterococcus faecium;
}

ERY: Erythromycin; ESBL: Extended-spectrum $\beta$-lactamase; EUSCAPE: Carbapenemase-Producing Enterobacteriaceae in Europe: FOX: Cefoxitin; IPM: Imipenem; KP-BSIs: Bloodstream infections caused by klebsiella pneumonia; KPN: Klebsiella pneumonia; LNZ: Linezolid; LVX: Levofloxacin; MDR: Multi-drug resistant; MEM: Meropenem; MIC: Minimum inhibitory concentration; MNO: Minocycline; MRSA: Methicillin-resistant S. aureus; PAE: Pseudomonas aeruginosa; PEN: Penicillin; PIP: Piperacillin; PMA: Stenotrophomonas maltophilia; SAM: Ampicillin-sulbactam; SAU: Staphylococcus aureus; SCN: Coagulase negative staphylococci; SPN: Streptococcus pneumonia; SXT: Trimethoprim Sulfamethoxazole; TCC: Ticarcillin-Clavulanate Acid; TEC: Teicoplanin; TZP: Piperacillin-tazobactam; VAN: Vancomycin; WHO: World Health Organization

\section{Acknowledgments}

We thank all of the members of HuBei Province bacterial resistance surveillance network. We thank Liwen Bianji, Edanz Editing China (www.liwenbianji.cn/ac), for editing the English text of a draft of this manuscript.

\section{Funding}

This work was supported by research grants from the National Mega Project on Major Infectious Disease Prevention (2017ZX10103005-007). The funders had no role in study design, data collection and analysis, decision to publish, or preparation of the manuscript.

\section{Availability of data and materials}

The datasets used and/or analysed during the current study are available from the corresponding author on reasonable request.

\section{Authors' contributions}

LT designed the study. ZZ did the statistical analysis. LT wrote the manuscript. ZS were in charge of the laboratory work. All authors read and approved the final manuscript.

\section{Authors' information}

Lei Tian and Ziyong Sun were from Department of Clinical Laboratory, Tongji Hospital, Tongji Medical College, Huazhong University of Science and Technology, Wuhan,Hubei Province, China. Zhen Zhang was from Department of Pharmacy, Tongji Hospital, Tongji Medical College, Huazhong University of Science and Technology, Wuhan,Hubei Province, China.

\section{Ethics approval and consent to participate}

The study protocol was approved by the Tongji Hospital ethics committee for research in health. The Tongji Hospital ethics committee also approved the waiver of informed consent to participate in this study due to its retrospective design. All patient data were anonymised prior to the analysis.

\section{Consent for publication}

Not applicable.

\section{Competing interests}

The authors declare that they have no competing interest.

\section{Publisher's Note}

Springer Nature remains neutral with regard to jurisdictional claims in published maps and institutional affiliations.

\section{Author details}

${ }^{1}$ Department of Clinical Laboratory, Tongji Hospital, Tongji Medical College, Huazhong University of Science and Technology, Wuhan, Hubei Province, China. ${ }^{2}$ Department of Pharmacy, Tongji Hospital, Tongji Medical College, Huazhong University of Science and Technology, Wuhan, Hubei Province, China.

Received: 21 February 2018 Accepted: 4 September 2018

Published online: 15 September 2018

\section{References}

1. Dat VQ, Vu HN, Nguyen The H, Nguyen HT, Hoang LB, Vu Tien Viet D, Bui

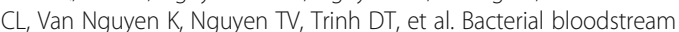
infections in a tertiary infectious diseases hospital in Northern Vietnam: 
aetiology, drug resistance, and treatment outcome. BMC Infect Dis. 2017; 17(1):493.

2. Huttunen R, Attman E, Aittoniemi J, Outinen T, Syrjanen J, Karki T, Lyytikainen O. Nosocomial bloodstream infections in a Finnish tertiary care hospital: a retrospective cohort study of 2175 episodes during the years 1999-2001 and 2005-2010. Infect Dis. 2015:47(1):20-6.

3. Musicha P, Cornick JE, Bar-Zeev N, French N, Masesa C, Denis B, Kennedy N, Mallewa J, Gordon MA, Msefula CL, et al. Trends in antimicrobial resistance in bloodstream infection isolates at a large urban hospital in Malawi (19982016): a surveillance study. Lancet Infect Dis. 2017;17(10):1042-52.

4. Wang S, Chen S, Feng W, Sun F, Wang Q, Zhu K, Song J. Clinica Characteristics of Nosocomial Bloodstream Infections in Neonates in Two Hospitals, China. J Trop Pediatr. 2018;64(3):231-6.

5. World Health Organization. Department of Communicable Disease, Surveillance and Response, Prevention of Hospital-acquired Infections. Geneva: World Health Organization; 2002. Publication WHO/CDS/CSR/EPH/ 2002.12

6. Levesque S, Bourgault AM, Galarneau LA, Moisan D, Doualla-Bell F, Tremblay C. Molecular epidemiology and antimicrobial susceptibility profiles of methicillin-resistant Staphylococcus aureus blood culture isolates: results of the Quebec Provincial Surveillance Programme. Epidemiol Infect. 2015; 143(7):1511-8.

7. Weinstein MP, Murphy JR, Reller LB, Lichtenstein KA. The clinical significance of positive blood cultures: a comprehensive analysis of 500 episodes of bacteremia and fungemia in adults. II. Clinical observations, with special reference to factors influencing prognosis. Rev Infect Dis. 1983;5(1):54-70.

8. Clinical and Laboratory Standards Institute. Performance Standards for Antimicrobial Susceptibility Testing, Twenty-sixth Informational Supplement, M100-S26. Wayne, PA: Clin Lab Stand Institute; 2016.

9. Clinical and Laboratory Standards Institute. Analysis and presentation of cumulative antimicrobial susceptibility test data, M39-A4. Wayne, PA: Clinical Lab Stand Institute; 2014.

10. Diekema DJ, Pfaller MA, Schmitz FJ, Smayevsky J, Bell J, Jones RN, Beach M, Group SP. Survey of infections due to Staphylococcus species: frequency of occurrence and antimicrobial susceptibility of isolates collected in the United States, Canada, Latin America, Europe, and the Western Pacific region for the SENTRY Antimicrobial Surveillance Program, 1997-1999. Clin Infect Dis. 2001;32(Suppl 2):S114-32.

11. Tian L, Zhu X, Chen Z, Liu W, Li S, Yu W, Zhang W, Xiang X, Sun Z. Characteristics of bacterial pathogens associated with acute diarrhea in children under 5 years of age: a hospital-based cross-sectional study. BMC Infect Dis. 2016;16:253.

12. Laupland KB, Gregson DB, Church DL, Ross T, Pitout JD. Incidence, risk factors and outcomes of Escherichia coli bloodstream infections in a large Canadian region. Clin Microbiol Infect. 2008;14(11):1041-7.

13. Kato K, Matsumura Y, Yamamoto M, Nagao M, Ito Y, Takakura S, Ichiyama S. Erratum to: Seasonal trend and clinical presentation of Bacillus cereus bloodstream infection: association with summer and indwelling catheter. Eur J Clin Microbiol Infect Dis. 2016;35(5):875-83.

14. Gagliotti C, Balode A, Baquero F, Degener J, Grundmann H, Gür D, Jarlier V, Kahlmeter G, Monen J, Monnet DL, et al. Escherichia coli and Staphylococcus aureus: bad news and good news from the European Antimicrobial Resistance Surveillance Network (EARS-Net, formerly EARSS), 2002 to 2009. Euro Surveill. 2011;16(14):pii/19834.

15. Hidron Al, Edwards JR, Patel J, Horan TC, Sievert DM, Pollock DA, Fridkin SK. National Healthcare Safety Network T, Participating National Healthcare Safety Network F: NHSN annual update: antimicrobial-resistant pathogens associated with healthcare-associated infections: annual summary of data reported to the National Healthcare Safety Network at the Centers for Disease Control and Prevention, 2006-2007. Infect Control Hosp Epidemiol. 2008;29(11):996-1011.

16. Schwaber MJ, Carmeli Y. Mortality and delay in effective therapy associated with extended-spectrum beta-lactamase production in Enterobacteriaceae bacteraemia: a systematic review and meta-analysis. J Antimicrob Chemother. 2007;60(5):913-20.

17. Rodriguez-Bano J, Picon E, Gijon P, Hernandez JR, Cisneros JM, Pena C, Almela M, Almirante B, Grill F, Colomina J, et al. Risk factors and prognosis of nosocomial bloodstream infections caused by extended-spectrum-betalactamase-producing Escherichia coli. J Clin Microbiol. 2010;48(5):1726-31.

18. de Kraker ME, Davey PG, Grundmann H, group Bs: Mortality and hospital stay associated with resistant Staphylococcus aureus and Escherichia coli bacteremia: estimating the burden of antibiotic resistance in Europe. PLoS Med 2011, 8(10):e1001104.

19. Leistner R, Gurntke S, Sakellariou C, Denkel LA, Bloch A, Gastmeier P, Schwab F. Bloodstream infection due to extended-spectrum beta-lactamase (ESBL)-positive K. pneumoniae and E. coli: an analysis of the disease burden in a large cohort. Infection. 2014;42(6):991-7.

20. Stewardson A, Fankhauser C, De Angelis G, Rohner P, Safran E, Schrenzel J, Pittet D, Harbarth S: Burden of bloodstream infection caused by extendedspectrum beta-lactamase-producing enterobacteriaceae determined using multistate modeling at a Swiss University Hospital and a nationwide predictive model. Infect Control Hosp Epidemiol 2013, 34(2):133-143.

21. Lin YT, Liu CJ, Fung CP, Tzeng CH. Nosocomial Klebsiella pneumoniae bacteraemia in adult cancer patients--characteristics of neutropenic and non-neutropenic patients. Scand J Infect Dis. 2011;43(8):603-8.

22. Nasa $P$, Juneja $D$, Singh $O$, Dang $R$, Singh $A$. An observational study on bloodstream extended-spectrum beta-lactamase infection in critical care unit: incidence, risk factors and its impact on outcome. Euro J Int Med. 2012;23(2):192-5.

23. Ben-David D, Kordevani R, Keller N, Tal I, Marzel A, Gal-Mor O, Maor Y, Rahav G. Outcome of carbapenem resistant Klebsiella pneumoniae bloodstream infections. Clin Microbiol Infect. 2012;18(1):54-60.

24. Zhao SY, Wang YC, Xiao SZ, Jiang XF, Guo XK, Ni YX, Han LZ. Drug susceptibility and molecular epidemiology of Escherichia coli in bloodstream infections in Shanghai, China, 2011-2013. Infect Dis. 2015;47(5):310-8.

25. Xiao SZ, Wang S, Wu WM, Zhao SY, Gu FF, Ni YX, Guo XK, Qu JM, Han LZ. The Resistance Phenotype and Molecular Epidemiology of Klebsiella pneumoniae in Bloodstream Infections in Shanghai, China, 2012-2015. Front Microbiol. 2017;8:250.

26. Durdu B, Hakyemez IN, Bolukcu S, Okay G, Gultepe B, Aslan T. Mortality markers in nosocomial Klebsiella pneumoniae bloodstream infection. SpringerPlus. 2016;5(1):1892.

27. De Laveleye M, Huang TD, Bogaerts P, Berhin C, Bauraing C, Sacre P, Noel A, Glupczynski Y. Multicenter study g: Increasing incidence of carbapenemaseproducing Escherichia coli and Klebsiella pneumoniae in Belgian hospitals. Eur J Clin Microbiol Infect Dis. 2017;36(1):139-46.

28. Widmer AF, Lakatos B, Frei R. Strict infection control leads to low incidence of methicillin-resistant Staphylococcus aureus bloodstream infection over 20 years. Infect Control Hosp Epidemiol. 2015;36(6):702-9.

29. You JHS, Choi KW, Wong TY, Ip M, Ming WK, Wong RY, Chan SN, Tse HT, Chau CTS, Lee NLS. Disease Burden, Characteristics, and Outcomes of Methicillin-Resistant Staphylococcus aureus Bloodstream Infection in Hong Kong. Asia Pac J Public Health. 2017;29(5):451-61.

30. Landrum ML, Neumann C, Cook C, Chukwuma U, Ellis MW, Hospenthal DR, Murray CK. Epidemiology of Staphylococcus aureus blood and skin and soft tissue infections in the US military health system, 2005-2010. JAMA. 2012; 308(1):50-9.

31. de Kraker ME, Jarlier V, Monen JC, Heuer OE, van de Sande N, Grundmann $\mathrm{H}$ : The changing epidemiology of bacteraemias in Europe: trends from the European Antimicrobial Resistance Surveillance System. Clin Microbiol Infect 2013, 19(9):860-868.

Ready to submit your research? Choose BMC and benefit from:

- fast, convenient online submission

- thorough peer review by experienced researchers in your field

- rapid publication on acceptance

- support for research data, including large and complex data types

- gold Open Access which fosters wider collaboration and increased citations

- maximum visibility for your research: over $100 \mathrm{M}$ website views per year

At $\mathrm{BMC}$, research is always in progress.

Learn more biomedcentral.com/submission 